\title{
Caracterização morfológica e classificação da superfície foliar de pimentas quanto à presença de tricomas e domácias
}

\author{
Cláudia Helena C Matos ${ }^{1,2}$; Angelo Pallini'; Cleide Maria F Pinto ${ }^{3}$; Madelaine Venzon ${ }^{3}$; Daniela DM \\ Rezende $^{1}$; Rita Cristina $P$ de Freitas ${ }^{1}$ \\ ${ }^{1}$ UFV, 36570-000 Viçosa-MG; 2UFRPE-UAST, Depto. de Biologia Animal, Entomologia; Fazenda Saco, s/n, 56900-000 Serra Talhada- \\ PE; c.helena@uast.ufrpe.br; ${ }^{3}$ EPAMIG-URZM, Vl. Gianetti 46, 36570-000 Viçosa-MG.
}

\begin{abstract}
RESUMO
O conhecimento das características morfológicas das plantas é de grande relevância para o estudo das interações entre plantas, herbívoros e inimigos naturais. Variações nessas características são determinantes na ocorrência, abundância e diversidade de organismos que exploram esse habitat. Caracterizou-se a superfície foliar de quatro espécies de pimentas do gênero Capsicum e elaborou-se uma proposta de classificação das folhas com base nos critérios de presença ou ausência, densidade e distribuição de tricomas para domácias, visando a utilização dessas informações em estudos de resistência de plantas a artrópodes. A contagem dos tricomas foi feita em uma área de $5 \mathrm{~cm}^{2}$, no terço médio das folhas, sendo contabilizados os tricomas do limbo, das nervuras e das domácias. As espécies de pimenta diferiram significativamente quanto à densidade de domácias e tricomas presentes na nervura e no limbo de suas folhas. Também foi observada diferença significativa na densidade total de tricomas/ $\mathrm{cm}^{2}$ e no número de tricomas por domácia. Das quatro espécies de pimenta avaliadas, apenas C. baccatum var. pendulum (pimenta com ardume variedade dedo-de-moça) não possui domácias nem tricomas em qualquer região de suas folhas. A maior densidade de domácias foi observada em C. baccatum var. praetermissum, espécie em que se observou as maiores densidades de tricomas em todas as regiões da folha e a única com densidade significativa de tricomas no limbo. As variações quanto à presença, distribuição e densidade de tricomas e domácias podem ser determinantes na ocorrência de ácaros predadores e fitófagos nas plantas. Com base na caracterização efetuada foi proposta uma escala de classificação da superfície foliar das plantas, variando de 1 a 5 , em função do número e da densidade de tricomas e domácias observados.
\end{abstract}

Palavras-chave: Capsicum spp., densidade de tricomas, domácia, defesa de plantas.

\begin{abstract}
Morphological characterization and surface classification of chili pepper leaves considering trichomes and domatia

The knowledge of morphological characteristics of plant leaves is important to study the interactions among plants, herbivores and natural enemies. Variations on such characteristics are determinant on the occurrence, abundance and diversity of organisms that exploit that habitat. The leaf surface of four Capsicum pepper species was characterized, focusing on the presence of trichomes and domatia. The objective is to identify possible mite resistance mechanisms on these plants. The classification key of pepper leaves was based on presence/absence, density and distribution of trichomes. The counting of trichomes was done in an area of $5 \mathrm{~cm}^{2}$, from the medium third of each leaf. We assessed the trichomes from the limb, ribs and domatia. The pepper species significantly differed on the density of domatia and trichomes present on the ribs and limbs of their leaves. A significant difference was observed on the total density of trichomes/ $\mathrm{cm}^{2}$ and on the number of trichomes/domatia. From the five Capsicum species evaluated, only C. baccatum var. pendulum (hot pepper, variety girl finger pepper) does not have domatia neither trichomes in any part of its leaves. The highest domatia density was found on C. baccatum var. praetermissum, which has the highest trichome density in all leaf parts and the only one with significant density of limb trichomes. The variation of the presence, distribution and density of trichomes and domatia may be determinant on the occurrence of herbivorous and predatory mites on plants. Such structures can act as a resistance mechanism against herbivore mites or may favor the presence of predatory and fungivorous mites due to provision of shelter sites. We propose here a surface classification scale of chili pepper leaves varying from 1 to 5 taking into account the number and density of observed trichomes and domatia.
\end{abstract}

Keywords: Capsicum spp., trichomes density, domatia, plant defense.

(Recebido para publicação em 30 de outubro de 2009; aceito em 15 de março de 2011)

(Received on October 30, 2009; accepted on March 15, 2011)

$\mathrm{O}$ conhecimento das características morfológicas das plantas é de grande relevância para o sucesso de estudos das interações entre plantas, herbívoros e inimigos naturais (Price, 1997). Espécies de um mesmo gênero de planta podem variar consideravelmente quanto às características das folhas, flores e frutos, e essas variações muitas vezes são determinantes da ocorrência, abundância e diversidade de organismos que as habitam (Petters, 2002).

Diversos estudos têm dado ênfase à importância dos mecanismos de defesa física das plantas na resistência contra insetos e ácaros (Lam \& Pedigo, 2001; Aragão et al., 2002; Paron \& Lara, 2005; Matos et al., 2009) e seus efeitos sobre os inimigos naturais (Krips et al., 1999; Styrsky et al., 2006). Tais características podem agir de forma positiva ou negativa sobre esses organismos, fazendo com que algumas espécies tenham preferência por residir em hábitats com determinadas características em relação a outras.

Muitas espécies de plantas apresentam em suas folhas tricomas ou pêlos que podem ter efeito negativo direto sobre os herbívoros (Almeida Cortez, 
2005). Tais estruturas podem atuar como uma defesa física (tricomas tectores), dificultando a mobilidade desses organismos sobre a superfície foliar, ou seja, funcionando como obstáculo, considerando a densidade, forma e tamanho que apresentam (Melo \& Silva Filho, 2002; Petters, 2002; Matos et al., 2009) ou ainda, como uma barreira química (tricomas glandulares), através da liberação de substâncias, podendo ter efeito imediato sobre a sua preferência alimentar, oviposição e sobrevivência, dentre outros aspectos (Krips et al., 1999; Mishalska, 2003; Simmons \& Gurr, 2005). Em alguns casos, podem atuar positivamente sobre esses organismos por disponibilizar refúgios que os protegem do ataque dos seus inimigos naturais (Mishalska, 2003).

Em relação aos predadores e parasitóides os efeitos da presença de tricomas também são variados. Estruturas como domácias, constituídas por cavidades ou tufos de pêlos (tricomas) nas junções entre nervura principal e secundária na face abaxial das folhas de diversas espécies de plantas (Lundströem, 1887), beneficiam a ocorrência e manutenção de ácaros predadores e fungívoros nas mesmas, disponibilizando locais de oviposição e abrigo contra condições adversas na superfície das folhas ou contra seus inimigos naturais, ou ainda, aumentando sua sobrevivência (Roda et al., 2000; Norton et al., 2001; Matos et al., 2004). Além disso, podem ter efeito negativo indireto sobre os herbívoros por propiciar a manutenção de seus inimigos naturais nas plantas, através da presença dessas estruturas (Matos et al., 2004; Mineiro et al., 2006).

O melhoramento genético visando a obtenção de cultivares resistentes a pragas e doenças vem sendo objeto de estudo de diversas pesquisas e tem abrangido várias culturas economicamente importantes (França et al., 2002; Paz Lima et al., 2004; Embrapa, 2006; Bento et al., 2007; Fernandes et al., 2009; Matos et al., 2009). Considerando o papel importante que as características morfológicas das plantas exercem tanto sobre os herbívoros, como sobre os inimigos naturais, é de grande importância o desenvolvimento de pesquisas que identifiquem como essas estruturas atuam na resistência contra esses organismos.

O cultivo de Capsicum spp. (pimentas e pimentões) no Brasil vem crescendo consideravelmente nos últimos anos, devido principalmente à versatilidade de aplicação, tanto no uso culinário, industrial, medicinal, como também no ornamental. Contudo, perdas decorrentes da incidência de pragas e doenças têm se tornado fonte de preocupação para os produtores (Amaral et al., 2001; Venzon et al., 2006; Pinto et al., 2007; Moura et al., 2010).

Estudos têm sido desenvolvidos visando identificar as espécies de Capsicum mais resistentes ao ataque de insetos e ácaros (Echer et al., 2002; Lima et al., 2003; Matos et al., 2009). Entretanto, no caso específico de ácaros, as informações são escassas e se resumem principalmente a listas das espécies possivelmente resistentes a esses organismos, sem relatar as prováveis causas dessa resistência (Echer et al., 2002; Lima et al., 2003). As plantas de Capsicum spp. variam consideravelmente quanto às características de suas folhas, havendo desde espécies com folhas glabras a espécies cobertas por tricomas (Metcalfe \& Chalk, 1950). Dessa forma, a resistência de determinadas espécies ao ataque de ácaros e insetos pode estar associada à presença e densidade dessas estruturas em suas folhas.

Este trabalho teve como objetivos caracterizar a superfície foliar de plantas de diferentes espécies de Capsicum quanto à presença de tricomas e domácias, elaborar uma classificação das folhas de Capsicum, de acordo com as características que estas possuem, visando sua utilização em estudos sobre interações entre ácaros fitófagos e predadores.

\section{MATERIAL E MÉTODOS}

Caracterização da superfície foliar de Capsicum spp. - As folhas utilizadas foram obtidas a partir de plantas das espécies Capsicum baccatum var. pendulum (pimenta com ardume, cultivar dedo-de-moça) Capsicum chinense (pimenta-bode), Capsicum frutescens (malagueta), Capsicum baccatum var. pendulum (pimenta sem ardume ou doce, cultivar chapéu-de-bispo) e Capsicum baccatum var. praetermissum (cumari) com seis meses de idade. De cada espécie foram amostradas 20 plantas adultas, retirando-se três folhas da região apical, de forma aleatória. As folhas foram acondicionadas, individualmente, em sacos de papel devidamente identificados, e levadas ao Laboratório de Acarologia da Universidade Federal de Viçosa. Foram coletadas folhas com área de aproximadamente $15 \mathrm{~cm}^{2}$, cuja medição foi feita utilizando-se o medidor portátil de área foliar (Delta-T Devices Ltd. Burwell, Cambridge, England).

A caracterização das folhas foi feita através da avaliação da distribuição e densidade de tricomas e domácias presentes por folha em cada espécie de Capsicum na região das nervuras e limbo. A contagem dos tricomas foi feita avaliando-se uma seção, com área de 5 $\mathrm{cm}^{2}$, do terço médio das folhas. Foram contabilizados os tricomas presentes na lâmina foliar e nas nervuras principal e secundárias, segundo metodologia de Goldman \& English-Loeb (2002). A caracterização das domácias foi feita segundo O'Dowd \& Wilson (1989). Foi contabilizada a densidade de domácias presentes por área foliar $\left(15 \mathrm{~cm}^{2}\right)$ e o número de tricomas presentes por domácia. Subseqüentemente, foi calculado o total de tricomas (limbo + nervura + domácias) presentes por folha.

Os dados obtidos foram submetidos à ANOVA e as médias comparadas pelo teste de Tukey a 5\% de probabilidade.

Classificação das espécies de Capsicum em função das características estudadas - De posse dos dados relativos à caracterização das domácias e tricomas de Capsicum spp. elaborou-se uma classificação para a arquitetura foliar das espécies estudadas, levando-se em consideração a ausência/presença de tricomas e domácias nas folhas, e a densidade de tricomas presentes nessas estruturas e na região do limbo e nervuras. Foi elaborada uma escala de 1 a 5 , organizada em ordem crescente da ocorrência das características supracitadas, ou seja, partindo inicialmente da espécie sem tricomas nem domácias (nota 1) até chegar à espécie com maior densidade 
de tricomas em todas as regiões da superfície foliar e nas domácias (nota 5) (Tabela 1). $\mathrm{O}$ aspecto geral das folhas de Capsicum spp. avaliadas, demostrando as características supracitadas, pode ser observado na figura 1.

\section{RESULTADOS E DISCUSSÃO}

Houve diferença significativa entre as espécies de pimenta quanto à densidade de domácias e tricomas presentes na nervura e no limbo de suas folhas. Também foi observada diferença significativa na densidade total de tricomas/ $\mathrm{cm} 2$ e no número de tricomas por domácia. Das espécies de Capsicum avaliadas apenas em $\mathrm{C}$. baccatum var. pendulum (pimenta com ardume, cultivar dedo-de-moça) não foram observadas domácias nem tricomas em quaisquer regiões de suas folhas, sendo esta espécie caracterizada como totalmente glabra (Figura 1A). A maior densidade de domácias foi observada em C. baccatum var. praetermissum (Folha coberta por tricomas, tanto no limbo como nas nervuras; domácias formando cavidades fechadas, obstruídas por tricomas (Fig. 1E)), não sendo observada diferença significativa na densidade dessas estruturas em $\mathrm{C}$. frutescens, C. chinense e C. baccatum var. pendulum (pimenta sem ardume ou doce, cultivar chapéu-de-bispo). A espécie C. frutescens apresentou suas folhas glabras com domácias, tricomas esparsos sem formar uma cavidade definida (Fig. 1B). Na espécie C. chinense foram observados folha glabra com domácias definidas formando cavidades parcialmente recobertas por tricomas (Fig. 1C) e, na espécie C. baccatum var. pendulum observou-se folha glabra com tricomas apenas na nervura central; domácias definidas formando cavidades fechadas, obstruídas por tricomas (Fig.1D). Por outro lado, todas as espécies de pimenta diferiram quanto ao número de tricomas presentes por domácia (Tabela 1).

$\mathrm{Na}$ classificação das folhas e suas ilustrações (Tabela 1, Figura 1), observa-se que C. baccatum var. praetermissum é a espécie cujas folhas mais se distinguem das demais, com as maiores densidades de tricomas em todas as regiões da folha, e sendo a única espécie com densidade significativa de tricomas no limbo, quando comparada às outras.

A variação observada quanto à presença, densidade e distribuição de tricomas nas folhas, deve ser considerada em pesquisas visando à identificação de espécies de Capsicum resistentes ao ataque de ácaros fitófagos e predadores. Tais características são de suma importância, uma vez que estudos têm demonstrado que as características morfológicas das plantas podem exercer forte influência sobre os aspectos biológicos e comportamentais desses organismos (Stavrinides \& Skirvin, 2003; Simmons \& Gurr, 2005).

Pesquisas têm demonstrado que estruturas das folhas como, por exemplo, os tricomas glandulares podem ter efeito negativo, tendo ação repelente sobre ácaros fitófagos (Aragão et al., 2002; Resende et al., 2002). Já em relação aos tricomas tectores é esperado que estes atuem como uma barreira física contra esses organismos; entretanto, poucos são os registros na literatura que dêem suporte a esta hipótese (Steinite \& Levinsh, 2003). Matos et al. (2009), avaliando a resistência de cultivares de Capsicum spp. ao ácaro-branco Polyphagotarsonemus latus (Acari: Tarsonemidae), observaram que os tricomas presentes nas cultivares estudadas atuaram como fator limitante ao desenvolvimento deste ácaro à medida que se apresentaram em maiores densidades e mais bem distribuídos na

Tabela 1. Caracterização e escala de classificação das folhas de cinco espécies de Capsicum, provenientes do banco de germoplasma da EPAMIG-CTZM e de espécies comerciais, quanto à distribuição e densidade de tricomas e domácias (characterization and classification scale of leaf surfaces of five Capsicum species, from the gene bank of EPAMIG-CTZM and from commercial species, assessing distribution and density of trichomes and domatia). Viçosa, UFV, 2010.

\begin{tabular}{|c|c|c|c|c|c|c|}
\hline \multirow[b]{3}{*}{ Nome cientifico (cultivar) } & \multicolumn{6}{|c|}{ Estruturas } \\
\hline & \multicolumn{4}{|c|}{ Tricomas (densidade por $5 \mathrm{~cm}^{2}$ ) } & \multicolumn{2}{|c|}{ Domácias } \\
\hline & Classe & Limbo & Nervura & $\begin{array}{c}\text { Total } \\
\text { (limbo+nervuras }+ \\
\text { domacias) } \\
\end{array}$ & $\begin{array}{l}\text { Densidade } \\
\text { por } 15 \mathrm{~cm}^{2}\end{array}$ & $\begin{array}{l}\text { N. de Tricomas } \\
\text { por domacias }\end{array}$ \\
\hline $\begin{array}{l}\text { C. baccatum var. pendulum }{ }^{1} \\
\text { (Dedo-de-moça) }\end{array}$ & 1 & $0,00 \mathrm{~B}$ & $0,00 \mathrm{D}$ & $0,00 \mathrm{E}$ & $0,00 \mathrm{C}$ & $0,00 \mathrm{E}$ \\
\hline C. frutescens $^{1}$ (Malagueta) & 2 & $0,00 \mathrm{~B}$ & $0,50 \mathrm{D}$ & $8,72 \mathrm{D}$ & $5,16 \mathrm{~B}$ & $8,22 \mathrm{D}$ \\
\hline C. chinense $^{2}$ (Bode) & 3 & $0,28 \mathrm{~B}$ & $45,83 \mathrm{C}$ & $73,61 \mathrm{C}$ & $5,36 \mathrm{~B}$ & $24,68 \mathrm{C}$ \\
\hline $\begin{array}{l}\text { C. baccatum var. pendulum }{ }^{1} \\
\text { (Chapéu-de-bispo) }\end{array}$ & 4 & $1,65 \mathrm{~B}$ & $179,05 \mathrm{~B}$ & $211,70 \mathrm{~B}$ & $5,40 \mathrm{~B}$ & $35,00 \mathrm{~B}$ \\
\hline $\begin{array}{l}\text { C. baccatum var. } \\
\text { praetermissum }^{2} \text { (Cumari) }\end{array}$ & 5 & $604,45 \mathrm{~A}$ & $235,85 \mathrm{~A}$ & $900,18 \mathrm{~A}$ & $8,15 \mathrm{~A}$ & $59,88 \mathrm{~A}$ \\
\hline
\end{tabular}

${ }^{1}$ Espécies comerciais; ${ }^{2}$ Espécies provenientes do banco de germoplasma da EPAMIG-CTZM. Médias seguidas de mesma letra na coluna não diferem a $5 \%$ de probabilidade pelo teste de Tukey $\left({ }^{1}\right.$ Commercial species; ${ }^{2}$ Species from the gene bank of EPAMIG-CTZM. Means followed by the same letter in column are not different according to Tukey test at $5 \%$ probability). 


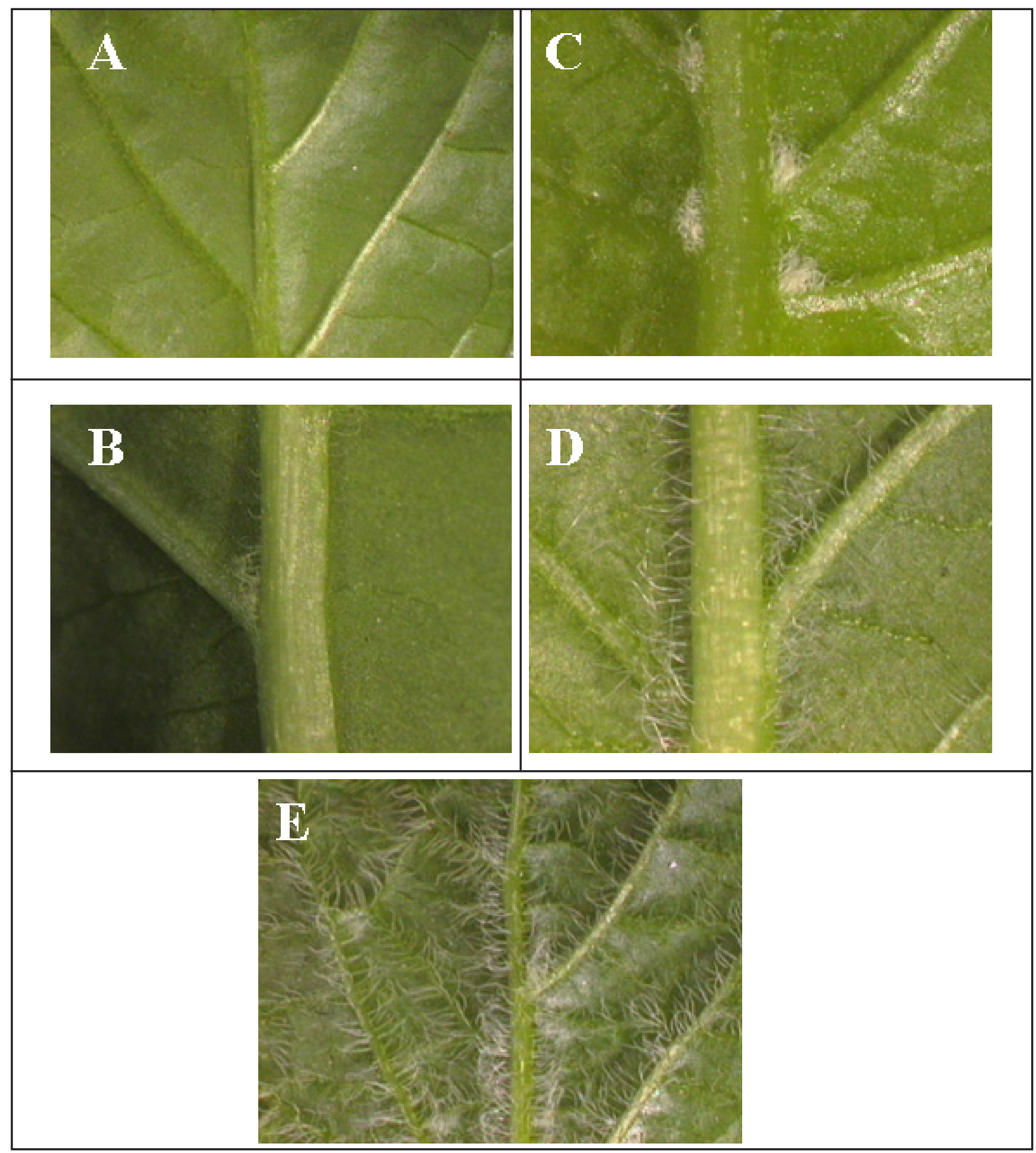

Figura 1. Aspecto geral das folhas de pimenta Capsicum quanto à presença/ausência de tricomas e domácias: (A) C. baccatum var. pendulum (pimenta com ardume, cultivar dedo-de-moça); (B) C. frutescens (pimenta malagueta); (C) C. chinense (pimenta-de-bode); (D) C. baccatum var. pendulum (pimenta sem ardume ou doce cultivar chapéu-de-bispo), (E) C. bacatum var. praetermissum (pimenta cumari) (general view of Capsicum pepper leaves showing presence/absence of trichomes and domatia: (A) C. baccatum var. pendulum, hot pepper, cultivar dedo-de-moça; (B) C. frutescens, smelling pepper; C. chinense, pimenta-de-bode; (D) C. baccatum var. pendulum, sweet pepper, cultivar chapéu-de-bispo (E) C. bacatum var. praetermissum, bird pepper). Viçosa, UFV, 2010.

superfície foliar, o que reforça a importância da realização de outros estudos que possibilitem avaliar o efeito dessas estruturas sobre aspectos biológicos e comportamentais de outras pragas associadas à cultura.

Em relação aos ácaros predadores, a maior parte dos estudos tem demons- trado que, de maneira geral, esses organismos se beneficiam da presença de determinadas estruturas, como tricomas e domácias (O’Dowd \& Pemberton, 
1998; Kreiter et al., 2002, Matos et al., 2004; Venzon et al., 2006). No caso das domácias, estas estruturas servem como local de oviposição para os predadores e de abrigo contra condições adversas na superfície da folha ou contra seus inimigos naturais (Norton et al., 2001; Matos et al., 2004), enquanto os tricomas na superfície foliar estão relacionados, dentre outros aspectos, com a retenção de alimento alternativo para esses ácaros, como pólen e esporos de fungos (Roda et al., 2003).

No que se refere às espécies de Capsicum, observou-se que estas variam quanto à resistência a ácaros fitófagos, como por exemplo, Polyphagotarsonemus latus (Acari: Tarsonemidae), importante praga desta cultura (Echer et al., 2002; Lima et al., 2003). Entretanto, as possíveis causas dessa variação não são explicadas. Diante disso, a partir da classificação das espécies de Capsicum quanto à superfície foliar, aqui apresentada, torna-se possível a realização de testes visando avaliar que papel os tricomas e as domácias podem exercer sobre esses organismos e seus inimigos naturais.

Observações de campo têm demonstrado, que plantas de Capsicum frutescens (pimenta malagueta) são bastante suscetíveis ao ataque de P. latus, o que representa um problema sério para os produtores de pimenta da Zona da Mata do estado de Minas Gerais (Cleide Maria Ferreira Pinto, comunicação pessoal). Essa espécie é descrita como tendo folhas com tricomas tectores curtos na superfície da epiderme (Adedeji et al., 2007), resultado corroborado pelo presente trabalho, uma vez que foi observada a presença dessas estruturas, em pequena densidade, nas domácias da espécie supracitada (Tabela 1), fato que pode estar contribuindo para sua suscetibilidade ao ataque deste ácaro. Assim, tomando como base essas informações, é esperado que espécies como C. baccatum var. praetermissum e C. baccatum var. pendulum (pimenta sem ardume ou doce, cultivar chapéu-de-bispo) apresentem maior resistência ao ataque desse ácaro, uma vez que suas folhas apresentam grandes densidades de tricomas e domácias (Tabela 1), entretanto, esta hipótese precisa ser testada.

Vale salientar ainda, que a presença e densidade de tricomas nas folhas das plantas podem variar consideravelmente com a idade das mesmas, com sua posição nas folhas (a região periférica geralmente é mais pilosa), com a superfície foliar (abaxial ou adaxial) e com a espécie de planta (Toledo et al., 2004). Assim, estudos voltados para a caracterização e avaliação da densidade e distribuição dessas estruturas nas plantas devem ser desenvolvidos tendo-se sempre o cuidado de se utilizar folhas totalmente expandidas, de maneira a se ter uma caracterização precisa, principalmente, no que diz respeito à distribuição dos tricomas nas mesmas, aspectos estes que devem ser considerados ao se avaliar o efeito dessas estruturas sobre os organismos que as habitam.

\section{AGRADECIMENTOS}

Ao CNPq e à CAPES pela concessão de bolsas de estudo e à EPAMIG pela disponibilidade de infra-estrutura de apoio ao desenvolvimento dos experimentos.

\section{REFERÊNCIAS}

ADEDEJI O; AJUWON OY; BABAWALE OO. 2007. Foliar epidermal studies, organographic distribution and taxonomic importance of trichomes in the family Solanaceae. International Journal of Botany 3: 276-282.

ALMEIDA CORTEZ J. 2005. Herbivoria e mecanismos de defesa vegetal. In: Nogueira RJMC; ARAÚJO EL; WILLADINO LG; CAVALCANTE UMT (Org.). Estresses ambientais: danos e benefícios em plantas. Recife. p. 389-396.

AMARAL DSLS; PALLINI A; PINTO CMF. 2001. Diagnóstico e recomendações técnicas para a cultura da pimenta na Zona da Mata Mineira. Viçosa: UFV. 39p (Boletim Técnico).

ARAGÃO CA; DANTAS BF; BENITES FRG. 2002. Efeito de aleloquímicos em tricomas foliares de tomateiro na repelência ao ácaro (Tetranychus urticae Koch.) em genótipos com teores contrastantes de 2-tridecanona. Acta Botânica Brasílica 16: 83-88.

BENTO CSB; SUDRE CP; RODRIGUES R; RIVA EM; PEREIRA MG. 2007. Descritores qualitativos e multicategóricos na estimativa da variabilidade fenotípica entre acessos de pimentas. Scientia Agraria 8: 149-156.

ECHER MM; FERNANDES MCA; RIBEIRO RLD; PERAQUI AL. 2002. Avaliação de genótipos de Capsicum para resistência ao ácaro branco. Horticultura Brasileira 20:
217-221.

EMBRAPA. 2006. Pimenta: cultivo. Disponível em: http://www.cnph.embrapa.br/pimenta/ cultivo.htm. Acessado em 25 de março de 2009.

FERNANDES MES; SILVADJH; FERNANDES FL; PICANÇO MC; GONTIJO PC; GALDINO TVS. 2009. Novos acessos de tomateiro resistentes à mosca-branca biótipo B. Pesquisa Agropecuária Brasileira 44: 1545-1548.

FRANÇA FH; RITSCHEL PS. 2002. Avaliação de acessos de batata-doce para resistência à broca-da-raiz, crisomelídeos e elaterídeos. Horticultura Brasileira 20: 79-85.

GOLDMAN K; ENGLISH-LOEB G. 2002. Influence of grape leaf topography on the predaceous mite Typhlodromus pyri. Geneva, Cornell University. 17p.

KREITER S; TIXIER MS; CROFT BA; AUGER P; BARRET D. 2002. Plants and leaf characteristics influencing the predaceous mite Kampimodromus aberrans (Acari: Phytoseiidae) in habitats surrounding vineyards. Environmental Entomology 31: 648-660.

KRIPS OE; KLEIJN PW; WILLEMS PEL; GOLS GJZ; DICKE M. 1999. Leaf hairs influence searching efficiency and predation rate of the predatory mite Phytoseiulus persimilis (Acari: Phytoseiidae). Experimental and Applied Acarology 23: 119-131.

LAM WK; PEDIGO LP. 2001. Effect of trichome density on soybean pod feeding by adult bean leaf beetles (Coleoptera: Chrysomelidae). Journal of Economic Entomology 94: 14591463.

LIMA MLP; MELO-FILHO PA; CAFÉ-FILHO AC. 2003. Colonização por ácaros em genótipos de pimentas e pimentões em cultivo protegido. Ciência Rural 33: 1157-1159.

LUNDSTRÖEM AN. 1887. Pflanzenbiologische studien II. Die Anpassungen der Pflanzen an Tiere. Domatienführende Pflanzen. In: MARIANI MJ. Les Caféiers. Paris: L' Université. p.1-88.

MATOS CHC; PALLINI A; CHAVES FF; GALBIATI C. 2004. Domácias do cafeeiro beneficiam o ácaro predador Iphiseiodes zuluagai Denmark \& Muma (Acari: Phytoseiidae)? Neotropical Entomology 33: 57-63.

MATOS CHC; PALLINI A; VENZON M; FREITAS RCP; REZENDE DDM; SCHOEREDER JH. 2009. Os tricomas de Capsicum spp. interferem nos aspectos biológicos do ácaro-branco Polyphagotarsonemus latus Banks (Acari: Tarsonemidae). Neotropical Entomology 38: 589-594.

MELO MO; SILVA-FILHO MC. 2002. Plantinsect interaction: an evolutionary arms race between two distinct defense mechanisms. Brazilian Journal of Plant Physiology 14: 71-81.

METCALFE CR; CHALK L. 1950. Anatomy of the dicotyledons: leaves, stem, and wood in relation to taxonomy with note on economic uses. London: Oxford University press, v. $2,1167 \mathrm{p}$. 
MINEIRO JLC; SATO ME; RAGAA; ARTHUR V; MORAES GJ; SARRETA FO; CARRIJO A. 2006. Diversidade de ácaros (Arachnida: Acari) em Coffea arabica L. cv. Mundo Novo, nos municípios de Jeriquara e Garça, Estado de São Paulo. Biota Neotropica 6 (2). Disponível em http://www.biotaneotropica.org.br/v6n2/ pt/fullpaper?bn01106022006+pt. Acessado em 07 de junho de 2010.

MISHALSKA K 2003. Clibing of leaf trichomes by eriophyid mites impedes their location by predators. Journal of Insect Behavior 16: 833-844.

MOURA MCCL; GONÇALVES LSA; SUDRÉ CP; RODRIGUES R; AMARAL JÚNIOR AT; PEREIRA TNS. 2010. Algoritmo de Gower na estimativa da divergência genética em germoplasma de pimenta. Horticultura Brasileira 28: 155-161.

NORTON A; ENGLISH-LOEB G; BELDEN E. 2001. Host plant manipulation of natural enemies: leaf domatia protect beneficial mites from insect predators. Oecologia 126: 535-542.

O’DOWD DJ; WILLSON MF. 1989. Leaf domatia and mites on Australasian plants: ecological and evolutionary implications. Biological Journal of the Linnean Society 37: 191-238.

O'DOWD DJ; PEMBERTON RW. 1998. Leaf domatia and foliar mite abundance in broadleaf deciduous forest of north Asia. American Journal of Botany 85: 70-78.

PARON MJFO; LARA FM. 2005. Relação entre tricomas foliares de genótipos de feijoeiro comum, Phaseolus vulgaris L. e resistência a Diabrotica speciosa Germar, 1824 (Coleoptera: Chrysomelidae). Ciência e Agrotecnologia 29: 894-898.

PAZ LIMA ML; LOPES CA; CAFÉ FILHO AC. 2004. Estabilidade da resistência de Capsicum spp. ao oídio em telado e casa de vegetação. Fitopatologia Brasileira 29: 519-525.

PETTERS PJ. 2002. Correlations between leaf structural traits and the densities of herbivorous insect guilds. Biological Journal of the Linnean Society 77: 43-65.

PINTO CMF; MOREIRA GR; CALIMAN FB; VENZON M; PAULA JUNIOR TJ. 2007. Pimenta (Capsicum spp.). In: PAULA JÚNIOR TJ; VENZON M (org). 101 Culturas: Manual de Tecnologias Agrícolas. Belo Horizonte: EPAMIG, p. 625-632.

PRICE PW. 1997. Insect Ecology. New York: John Wiley \& Sons, 874p.

RESENDE JTV; MALUF WR; CARDOSO MG; NELSON DL; FARIA MV. 2002. Inheritance of acylsugar contents in tomatoes derived from an interspecific cross with the wild tomato Lycopersicon pennellii and their effect on spider mite repellence. Genetics and Molecular Research 1: 106-116.

RODA AL; NYROP JP; DICKE M; ENGLISHLOEB G. 2000. Trichomes and spider mite webbing protect predatory mite eggs from intraguild predation. Oecologia 125: 428-435.

RODA AL; NYROP JP; DICKE M; ENGLISHLOEB G. 2003. Leaf pubescence mediates the abundance of non-prey food and the density of the predatory mite Typhlodromus pyri. Experimental and Applied Acarology 29: 193-211.

SIMMONS AT; GURR GM. 2005. Trichomes of Lycopersicon species and their hybrids: effects on pests and natural enemies. Agricultural and Forest Entomology 7: 265-276.

STAVRINIDES MC; SKIRVIN DJ. 2003. The effect of chrysanthemum leaf trichome density and prey spatial distribution on predation of Tetranychus urticae (Acari: Tetranychidae) by Phytoseiulus persimilis. Bulletin of Entomological Research 93: 343-350.

STEINITE I; LEVINSH G. 2003. Possible role of trichomes in resistance of strawberry cultivars against spider mite. Acta Universitatis Latviensis 662: 59-65.

STYRSKY JD; KAPLAN I; EUBANKS MD. 2006. Plant trichomes indirectly enhance tritrophic interactions involving a generalist predator, the red imported fire ant. Biological Control 36: 375-384.

TOLEDO MGT; ALQUINI Y; NAKASHIMA T. 2004. Caracterização anatômica das folhas de Cunila microcephala Benth. (Lamiaceae). Revista Brasileira de Ciencias Farmacêuticas 40: 487-493.

VENZON M; MATOS CHC; ROSADO MC; PALlinI A; SANTOS IC. 2006. Pragas associadas à cultura da pimenta e estratégias de manejo. Informe Agropecuário 27:75-86. 